\title{
miR-2I Induces Chemoresistance in Ovarian Cancer Cells via Mediating the Expression and Interaction of CD44v6 and P-gP
}

This article was published in the following Dove Press journal: OncoTargets and Therapy

\author{
Yanqing Wang ${ }^{\mathrm{l}} *$ \\ Gantao Chen ${ }^{2, *}$ \\ Fangfang Dai ${ }^{1}$ \\ Li Zhang' \\ Mengqin Yuan' \\ Dongyong Yang' \\ Shiyi Liu' \\ Yanxiang Cheng'
}

'Department of Obstetrics and Gynecology, Renmin Hospital of Wuhan University, Wuhan, Hubei 430060,

People's Republic of China; ${ }^{2}$ Department of Gastroenterology, Third People's Hospital of Xiantao in Hubei Province, Wuhan 433000, People's Republic of China

*These authors contributed equally to this work
Correspondence: Yanxiang Cheng Department of Obstetrics and Gynecology, Renmin Hospital of Wuhan University, 99 Zhang Zhidong Road, Wuhan, Hubei 430060, People's Republic of China

Email yanxiangCheng@whu.edu.cn
Background: Ovarian cancer (OC), a representative female reproductive system tumor, is one of the most malignant tumors in female. The most important reason for its poor prognosis is because of its high rate of chemotherapy resistance.

Results: This study aims to explore the effects of miR-21 on the chemotherapy resistance of OC cells. The functions of miR-21 on proliferation, migration and invasion of OC cells were assessed by transwell, clonal formation and CCK8 assay. Expression levels of miR-21, P-gp and CD44v6 in SKOV3 (cisplatin sensitive) cells and SKOV3/DDP (cisplatin resistant) cells were detected by quantitative reverse transcription-polymerase chain reaction (qRT-PCR) and Western blotting. Si-CD44v6 was transfected into OC cells to detect the influence on P-glycoprotein (P-gp) expression. Immunofluorescence was used to detect the localization of CD44v6 and P-gp in cell. Co-immunoprecipitation was used to detect the relationship between CD44v6 and P-gp. Results showed that miR-21 expression in cisplatin-resistant SKOV3/DDP cells was significantly higher than that in SKOV3 cells, at the same time, cells proliferation, as well as invasion and migration ability were enhanced after the miR-21 mimics transfected into SKOV3 cisplatin-sensitive cells. Furthermore, miR-21 expression level affected the CD44v6 and P-gp expression. Immunofluorescence and co-immunoprecipitation showed that CD44v6 and P-gp protein could interact.

Conclusion: In conclusion, the high miR-21 expression level could increase the proliferation, invasion, and migration ability of OC cells. And the interaction of CD44v6 and P-gp may mediate miR-21 involvement in chemotherapy resistance of OC cells.

Keywords: ovarian cancer, chemotherapy resistance, CD44v6, P-glycoprotein

\section{Introduction}

Ovarian cancer (OC), the fifth most common tumor among women, which has the highest rate of mortality in female reproductive system tumors in the worldwide. Five-year survival rate for patients with $\mathrm{OC}$ is less than $50 \% .^{1-3}$ According to the statistics, in 2018, there are approximately 15,000 deaths were caused by OC in the United States. ${ }^{4}$ And the OC caused around 22,000 deaths in China every year., Despite initially effective platinum- and taxane-based treatment, however, the outcome of $\mathrm{OC}$ patients is not optimistic for $\mathrm{OC}$ have high chemotherapy resistance rate and recurrence rate, which result the dead of patients. ${ }^{7}$ Chemotherapy of epithelial ovarian carcinoma using platinum-based drugs such as cisplatin is one of the first-line treatment modalities that is used in combination with other treatments. $^{8}$ At the beginning of chemotherapy, the relapsed OC shows hopeful 
improvement. However, after the completion of the treatment course, the tumor progression-free interval becomes shorter until the disease becomes incurable, in large part due to the development of chemoresistance potential in the OC cells. ${ }^{9}$ Therefore, to understanding the mechanism of chemotherapy resistance and identifying novel targets with prognostic, diagnostic, and therapeutic are of great value. As we all known that MicroRNAs (miRNAs) can downregulate the expression of genes via either mRNA degradation or inhibition of their translation as a type of noncoding RNAs. ${ }^{10}$ The association between cancer development and aberrant miRNA regulation have been studied by many researchers. ${ }^{11-13}$ And they found that miRNAs function as either tumor suppressors or promoters in carcinogenesis. ${ }^{14}$ It has been proven that dysregulation of many genes and various microRNAs (miRNAs or miRs) are associated with the occurrence of different types of malignancy. ${ }^{15}$ In the study of non-small cell lung cancer, inhibition of miR-21 expression can enhance the drug sensitivity of lung cancer-resistant cisplatin cells A549/ DDP to DDP, while overexpression of miR-21 can cause A549 cells to be resistant to chemotherapy drugs. The occurrence of drugs. ${ }^{16}$ In recent years, many researchers have discovered microRNA 21 (miR-21) were closely related to OC chemotherapy. ${ }^{17,18}$ Echevarría et al found that miR-21 can regulate OC chemotherapy via JNK-1/cJun pathway. ${ }^{18}$ Yang et al found that miR-21 can suppress the phosphate and tensin homolog (PTEN) transcript, which may activate the Akt pathway, and thus inducing chemoresistance of OC. ${ }^{19}$

P-glycoprotein (P-gp) which is a transmembrane protein that can efflux drugs, and reduce the intracellular bioavailability of drugs, thereby reducing the efficacy of drugs against cancer. Previous research has demonstrated that over-expression of the multidrug resistance 1 (MDR1) gene which encodes P-gp in several types of cancer is mainly contribute to drug resistance. And studies have demonstrated that miR-21 can induce OC cancer by regulating P-gp expression partly. ${ }^{20} \mathrm{CD} 44$, type I transmembrane receptor glycoprotein, is an important surface receptor for hyaluronic acid in the extracellular matrix, having CD44s and CD44v two kinds of forms. CD44V is expressed only in tumor cells and a few in normal epithelial cells. CD44 can involve a variety of biological processes, such as participating in lymphocyte homing activation, cytoskeletal anchoring, cell-cell/matrix adhesion, activation of multiple strips signals transduction pathways. ${ }^{21}$ Ravindranath et $\mathrm{al}^{22}$ demonstrated that CD44 increases P-gp mediated drug resistance. Miletti et $\mathrm{al}^{23}$ demonstrated that P-gp and CD44 are co-regulated, coimmunoprecipitated, and due to their interaction promotes tumor cell migration and invasion ability. However, in OC, the relationship between miR-21 and CD44v6 and P-gp is remain unclear. In present study, we took use of miR-21 mimics/inhibitors and siRNA-CD44v6 to explore the relationship between miR-21 and chemoresistance, and the relationship between miR-21, CD44v6 and P-gp in OC cells.

\section{Materials and Methods Cell Culture}

SKOV3 human ovarian carcinoma cells were obtained from the Shanghai Cell Bank of the Chinese Academy of Sciences (Shanghai, China). SKOV3 cells were cultivated in DMEM/F12 medium (SH30023.01, Hyclone) with 10\% FBS, $100 \mathrm{U} / \mathrm{mL}$ penicillin and $100 \mu \mathrm{g} / \mathrm{mL}$ streptomycin (Sangon Biotech Co., Ltd., Shanghai, China) at $37^{\circ} \mathrm{C}$ in a $5 \% \mathrm{CO}_{2}$ incubator.

\section{Cell Transfection}

The Negative control and miR-21 mimics/inhibitors were purchased from Sigma-Aldrich (Shanghai, China). SKOV3 and SKOV3/DDP cells were divided into negative control (NC) group which is to eliminate non-sequence specific effect and the mimics/inhibitors group to measure the transfection efficiency of miR-21, respectively. The negative control group in our study was transfected with empty vector. SKOV3 and SKOV3/DDP cells $\left(4 \times 10^{5}\right.$ cells/well) were respectively seeded into six-well plates, then the miR-21 or the NC mimics/inhibitors were transfected into cells with Lipofectamine ${ }^{\circledR} 2000$ (Thermo Fisher Scientific, Inc., Waltham, MA, USA) according to the manufacturer's recommendation.

\section{In vitro Silencing}

Cells were plated in 96-well plates with DMEM/F12 medium without antibiotics cultured for $24 \mathrm{~h}$ before transfection. The cells were transfected with signal (concentration: $50 \mathrm{nM}$ ), purchased from QIAGEN, targeting CD44v6: 5'GCCAACATTCATTCAATAC-3', and Lipofectamine 2000 (11668019, Invitrogen) according to the manufacturer's protocol. The cells were transfected once more after $24 \mathrm{~h}$ and harvested the next day. Scrambled siRNA was used as negative controls. 


\section{Clonal Formation Assay}

The cells were seeded in a six-well plate at a cell density of 1000 cells/well, and then the six-well plates were placed in a $37^{\circ} \mathrm{C}, 5 \% \mathrm{CO}_{2}$ incubator for 2 weeks. The cells were fixed with $4 \%$ polymethanol for $20 \mathrm{~min}$. Subsequently, dilute into crystal violet working solution with physiological saline and crystal violet mother liquor at a ratio of 2:9. The crystal violet dye solution was added to wells for $30 \mathrm{~min}$ and washed twice with PBS. Place a photo under the microscope and count the number of clones larger than 10 cells. Calculated clone formation rate: clone formation rate $(\%)=$ number of clones/number of cells inoculated $* 100 \%$.

\section{Transwell Assay}

For invasion assay, the matrigel and the medium were diluted 1:3, and $50 \mu \mathrm{L}$ was added to the transwell chamber, which was dried in an incubator for use. The cells were made into a suspension of $10^{5}$ cells $/ \mathrm{mL}$, and $1 \mathrm{~mL}$ of the cell suspension was centrifuged at $10,000 \mathrm{~g}$ for $5 \mathrm{~min}$, and the supernatant was discarded. Then, add $1 \mathrm{~mL}$ of medium without serum, blow evenly, and take $200 \mu \mathrm{L}$ of the cell suspension into a transwell chamber. $500 \mu \mathrm{L}$ of medium containing $10 \%$ FBS was added to a 24-well plate. Incubate for $48 \mathrm{~h}$ at $37^{\circ} \mathrm{C}$ in a $\mathrm{CO}_{2}$ (content $5 \%$ ) incubator. The chamber was taken out, the medium was washed away with PBS, the glue and cells in the upper chamber were wiped clean with a cotton swab, $0.01 \%$ crystal violet stained for $10 \mathrm{~min}$, the surface crystal violet was washed away, and the non-cell inoculation side was photographed under an inverted microscope (IX51; OLYMPUS). The average values of 5 randomly selected fields were used. For migration assay, there is no need to add the Matrigel and the medium to the transwell chamber. The remaining steps are the same as before.

\section{CCK8}

SKOV3 cells $(4,000$ cells/well) were cultured in 96-well plates overnight. Subsequently, SKOV3 and SKOV3/DDP cells were treated with different concentrations of cisplatin $(0,0.25,0.5,1,2,4$ and $6 \mu \mathrm{M})$ for $0,2,4,12$ and $24 \mathrm{~h}$. At the same time, SKOV3 and SKOV3/DDP cells were treated with cisplatin (the concentration at $\mathrm{IC}_{50}$ at $24 \mathrm{~h}$ ). At last, $10 \mu \mathrm{L}$ CCK $8(5 \mathrm{mg} / \mathrm{mL})$ solution was added to each well and incubated at a temperature of $37^{\circ} \mathrm{C}$ in a humidified atmosphere of $5 \% \mathrm{CO}_{2}$ for $1.5 \mathrm{~h}$. Then, the absorbance of the plate was measured at $490 \mathrm{~nm}$ using Automatic microplate reader (Perkin Elmer, USA).

\section{Western Blot}

SKOV3 and SKOV3/DDP cells $\left(1 \times 10^{6}\right.$ cells/well $)$ were cultured in 6-well plates overnight at $37^{\circ} \mathrm{C}$ in a $5 \% \mathrm{CO}_{2}$ incubator. Then, the SKOV3 cells were treated with lysis buffer with protease and phosphatase inhibitors (Beyotime Institute of Biotechnology) for $60 \mathrm{~min}$ and centrifuged at $12,000 \times \mathrm{g}$ for $10 \mathrm{~min}$ at $4^{\circ} \mathrm{C}$. Protein concentration was measured by BCA protein assay kit (AS1086, ASPEN). 10 $\mu \mathrm{g}$ of total protein lysate was electrophoresed in $10 \%$ SDS-polyacrylamide gels and the separated proteins in gels were transferred to PVDF membranes (EMD Millipore, Billerica, MA, USA). The membranes were blocked with $5 \%$ milk blocking solution for $2 \mathrm{~h}$ at room temperature. The blocking solution was removed and the diluted primary antibody anti-P-gp (abs131273; 1:500; Absin Bioscience Inc. China), anti-CD44v6 (ab78960; 1:500; abcam) and anti-GAPDH (ab37168; 1:1,000; Abcam, UK) was added overnight at $4^{\circ} \mathrm{C}$. Then, wash three times with TBST for $5 \mathrm{~min}$ each time. Add the diluted secondary antibody (HRP-Goat anti Rabbit, AS1107, ASPEN; HRP-Goat anti-Mouse, AS1106, ASPEN), incubate at room temperature for $2 \mathrm{~h}$, and wash four times with TBST on a shaker at room temperature for 5 min each time. The freshly prepared ECL mixed solution was added dropwise to the protein side of the membrane, and exposed in a dark room. The AlphaEaseFC software processing system analyzes the optical density values of the target bands.

\section{Quantitative Reverse Transcription- Polymerase Chain Reaction (qRT-PCR)}

Aspirate the medium in the cell culture plate. The residual medium was washed by adding $1 \mathrm{~mL}$ of PBS solution, and the PBS solution was pipetted. Then, $1 \mathrm{~mL}$ of Trizol reagent (Cat.no 15596-026, Invitrogen ${ }^{\mathrm{TM}}$ ) was added and the cells were thoroughly pipetted into Trizol. $250 \mu \mathrm{L}$ chloroform was added, and allowed to stand on ice for 5 $\mathrm{min}$. The mixture was centrifuged at $10,000 \mathrm{~g}$ for $10 \mathrm{~min}$ at $4^{\circ} \mathrm{C}$. Pipette $400 \mu \mathrm{L}$ of the supernatant into $1.5 \mathrm{~mL} \mathrm{EP}$ tube, add equal volume of $4^{\circ} \mathrm{C}$ pre-cooled isopropanol, mix by inversion, and let stand at $-20^{\circ} \mathrm{C}$ for $15 \mathrm{~min}$. Solution was centrifuged at $10,000 \mathrm{~g}$ for $10 \mathrm{~min}$ at $4^{\circ} \mathrm{C}$, pour off the liquid, add $1 \mathrm{~mL} 4^{\circ} \mathrm{C}$ pre-cooled $75 \%$ ethanol, reverse several times, wash the RNA precipitate, then 
Table I Primer Sequence

\begin{tabular}{|c|c|c|}
\hline \multicolumn{2}{|c|}{ Primer Name } & \multirow{3}{*}{$\begin{array}{l}\text { Primer Sequence } \\
5^{`} \text { - CATCATCCCTGCCTCTACTGG-3`'} \\
5^{\prime} \text { - GTGGGTGTCGCTGTTGAAGTC-3` }\end{array}$} \\
\hline GAPDH & $\mathrm{F}$ & \\
\hline & $\mathrm{R}$ & \\
\hline \multirow[t]{2}{*}{ CD44V6 } & $\mathrm{F}$ & 5’- GCCTTTGATGGACCAATTACC-3` \\
\hline & $\mathrm{R}$ & 5'- TCATTCCTATTGGTAGCAGGGA-3’' \\
\hline \multirow[t]{2}{*}{ P-gp } & $\mathrm{F}$ & 5`- CCAGAGGGCATGTTCATAGTT-3` \\
\hline & $\mathrm{R}$ & 5'- ACTGTCTTTCCTCGCTCAATAC-3` \\
\hline \multirow[t]{4}{*}{ U6 } & RT & $5^{`}$-AACGCTTCACGAATTTGCGT-3` \\
\hline & Primer & \\
\hline & $\mathrm{F}$ & 5'-CTCGCTTCGGCAGCACAT-3’ \\
\hline & $\mathrm{R}$ & $5^{`}$-AACGCTTCACGAATTTGCGT-3’ \\
\hline \multirow[t]{3}{*}{ hsa-miR-2I } & RT Primer & 5'-CTCAACTGGTGTCGTGGAGTCGGCAATTCAGTTGAGTCAACATC-3’ \\
\hline & $\mathrm{F}$ & 5`-TGGGCTTATCAGACTGATGTTGA-3` \\
\hline & $\mathrm{R}$ & 5`- CTCAACTGGTGTCGTGGAGTC-3` \\
\hline
\end{tabular}

centrifuged at $10,000 \mathrm{~g}$ for $10 \mathrm{~min}$ at $4^{\circ} \mathrm{C}$. Subsequently, pour off the liquid, dry for a few minutes, add $10 \mu \mathrm{L}$ RNase-Free Water to dissolve RNA. Total RNA (1 $1 \mu \mathrm{g})$ was reversed transcribed into to cDNA using PrimeScript RT Master Mix (Takara Bio, Inc., Otsu, Japan). qPCR was conducted using the StepOne ${ }^{\text {TM }}$ Real-Time PCR (Life technologies). A Bulge-Loop miRNA qRT-PCR kit (Invitrogen; Thermo Fisher Scientific, Inc.) was used to detect the relative expression of miRNA-21. The primers used were in Table 1.

\section{Co-Immunoprecipitation}

The protein in the cells is extracted by cell lysate and protease inhibitor. The sample protein concentration was detected by a BCA protein concentration assay kit. Add 20 $\mu \mathrm{L}$ magnetic beads to the appropriate volume of IP lysate in a centrifuge tube, and wash on a vertical shaker for $5 \mathrm{~min}$. Place the centrifuge tube on a magnetic stand for 5-10 s, aspirate the supernatant (try to avoid touching the beads). $1.0 \mu \mathrm{g}$ of IgG and $20 \mu \mathrm{L}$ of magnetic beads were added to the cell lysate supernatant or the tissue lysate supernatant, and incubated at $4^{\circ} \mathrm{C}$ for $30 \mathrm{~min}$ with shaking. The same amount of the above cell lysate was supernatanted into a 1.5 $\mathrm{mL}$ centrifuge tube, and $1-10 \mu \mathrm{L}(0.2-2 \mu \mathrm{g})$ of the antibody was added to precipitate one of the two interacting proteins, and the same amount of $\mathrm{IgG}$ was added as a negative control at $4^{\circ} \mathrm{C}$. Incubate for $1 \mathrm{~h}$. Add $20 \mu \mathrm{L}$ of magnetic beads, gently rub with your fingers, and incubate overnight at $4^{\circ} \mathrm{C}$; place the centrifuge tube on a magnetic stand for 5-10 s, and aspirate the supernatant. Add the appropriate volume of
Washing buffer to the centrifuge tube and place it on a vertical shaker for 3 minutes. Place the tube on the magnetic stand for 5-10 s and aspirate the supernatant (to avoid touching the beads). Repeat 3 times. After the last wash, carefully discard the supernatant, add an appropriate volume of 2 x SDS thioglycol-containing loading buffer, boil for $10 \mathrm{~min}$, and store at $-20^{\circ} \mathrm{C}$. Apply SDS-PAGE electrophoresis. Transfer film. Incubate the antibody.

\section{Immunofluorescence}

SKOV3 cells were cultured on glass coverslips until $80 \%$ confluent. Then, fixed with $4 \%$ formaldehyde solution and blocked with $5 \%$ bovine serum albumin (BSA; 10735078001; Roche; Switzerland) for $30 \mathrm{~min}$ at room temperature. Next, the cells were incubated with rabbit polyclonal anti-P-gp antibody (1:100; abcam, UK; cat. no. ab235954) overnight at $4^{\circ} \mathrm{C}$ and Fluorescein (FITC)-conjugated Affinipure Goat Anti-Rat IgG (H+L) (1:100; Wuhan Sanying Biotechnology Co., Ltd., China; cat. no. SA00003-11) for $1 \mathrm{~h}$ at $37^{\circ} \mathrm{C}$. The cells were then Observed by Fluorescence microscope (Olympus, IX51) following DAPI staining (1:1,000; cat. no. AS1075; Wuhan Aspen Biotechnology Co., Ltd., China).

\section{Data Statistics}

Data were described as mean $\pm \mathrm{SEM}$. Analyses were conducted with IBM SPSS Statistics (version 20.0) software, using the unpaired Student's $t$-test or one-way analysis of variance (ANOVA). And the post hoc test is Bonferroni. ${ }^{*} p<0.05$ were considered statistically significant. 


\section{Results}

\section{The $\mathrm{IC}_{50}$ and miR-2I Expression Level in OC Cells}

First, we tested the $\mathrm{IC}_{50}$ values of SKOV3, SKOV3/DDP cells and A2780, A2780/DDP cells. The result suggests that the $\mathrm{IC}_{50}$ value is higher in SKOV3/DDP cells than that in SKOV3 cells (Figure 1A, $P<0.01$ ). And the result is similar in A2780 and A2780/DDP cells (Figure 1A, $P<0.05$ ). Subsequently, to identified miR-21 expression in OC cells, we determined miR21 expression in SKOV3 and SKOV3/DDP cells, A2780 and A2780/DDP cells. miR-21 was up-regulated in SKOV3/DDP and A2780/DDP cells (Figure 1B and C). This result suggests that miR-21 is highly expressed in $\mathrm{OC}$ resistant cells, which may play a role in $\mathrm{OC}$ chemotherapy resistance.

\section{miR-2I Promotes Chemotherapy Resistance in OC Cells}

We next assessed the effect of miR-21 in cell growth, proliferation, invasion, and migration. For this set of experiments, we took use of miR-21 mimics and corresponding negative control transient transfection in SKOV3 and A2780 cells, miR-21 inhibitors, and corresponding negative control transient transfection in SKOV3/DDP and A2780/DDP cells. We confirmed the down-regulation of miR-21 expression by the miR-21 inhibitors and the up-regulation of miR-21 expression by the miR-21 mimics (Figure 1B and C). Figure 2A and B shows the cell viability time effect curve under the cisplatin treatment $\left(\mathrm{IC}_{50}\right)$. In SKOV3 + miR-21 mimics group, cell viability was significantly higher than that in SKOV3 + miR-21 negative control group (Figure $2 \mathrm{~A}, P<0.05$ ). And in the SKOV3/DDP + miR-21 inhibitors group, cell viability was decreased than that in SKOV3/DDP + miR-21 negative control group (Figure $2 \mathrm{~A}, P<0.05$ ). Results in A2780 cell lines are similar with that in SKOV3 cells (Figure $2 \mathrm{~B}, P<0.05$ ).

\section{Effect of miR-2I in OC Cells Growth, Proliferation, and Invasion}

To demonstrate whether miR-21 can lead to the cisplatin resistance of OC cells, we transient transfected miR-21 mimics and inhibitor in SKOV3 and SKOV3/DDP cells. Thus, we assessed in treatment with the same concentration and the same time length of cisplatin solution, clonal proliferation, migration, and invasion ability of each group of cells (Figures 3 and 4). In a colony formation assay, compared to the NC-inhibitor transfected cells (Figure 3A and $\mathrm{B}$ ), the number of colonies formation were reduced significantly in the transient transfection of miR-21 inhibitor in SKOV3/DDP cells (Figure 3, $p<0.05$ ). Transwell assay confirmed that $50 \mathrm{nM}$ of miR-21 inhibitors reduced obviously $(50 \%, p<0.05)$ in the migration and invasion ability of SKOV3/DDP cells compared that in NC inhibitors (Figure 4). This result demonstrates that high levels of miR-21 are relative to the metastatic potential of cisplatin resistant OC cells, and that miR-21 is a potential target to overcome the cisplatin resistance of highly metastatic OC cells.

\section{CD44v6 and P-gp Expression Can Be Affected by miR-2I}

We determined P-gp and CD44v6 expression in SKOV3 and SKOV3/DDP cells. CD44v6 was up-regulated in
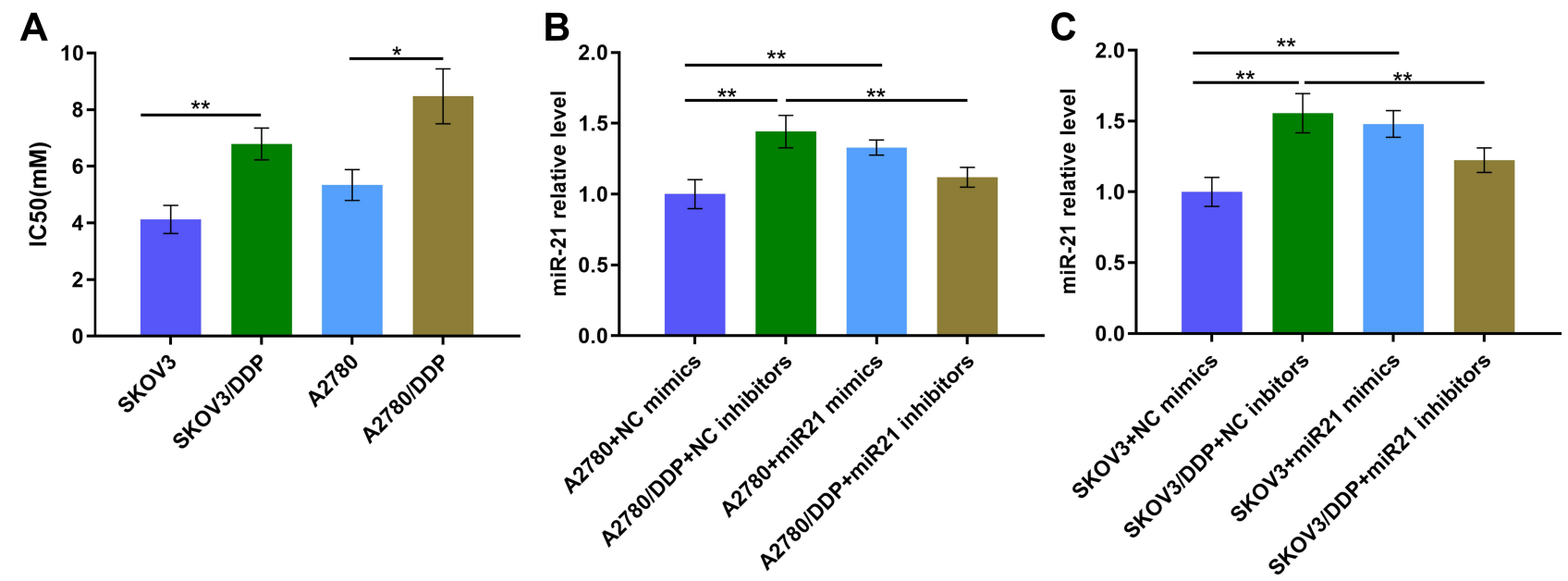

Figure I (A) The IC 50 value of different cells. (B) miR-2I expression level of A2780 cells transfected in miR2I mimics/inhibitors/NC by qRT-PCR. (C) miR-2I expression level of SKOV3 cells transfected in miR2I mimics/inhibitors NC by qRT-PCR. $* P<0.05$; $* * P<0.01$. 
A

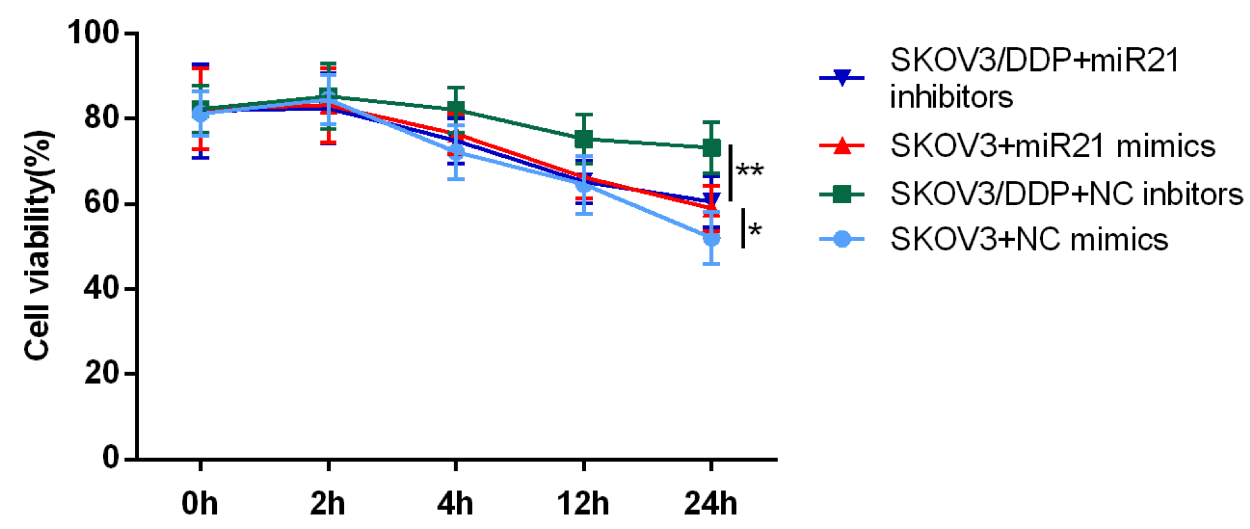

B

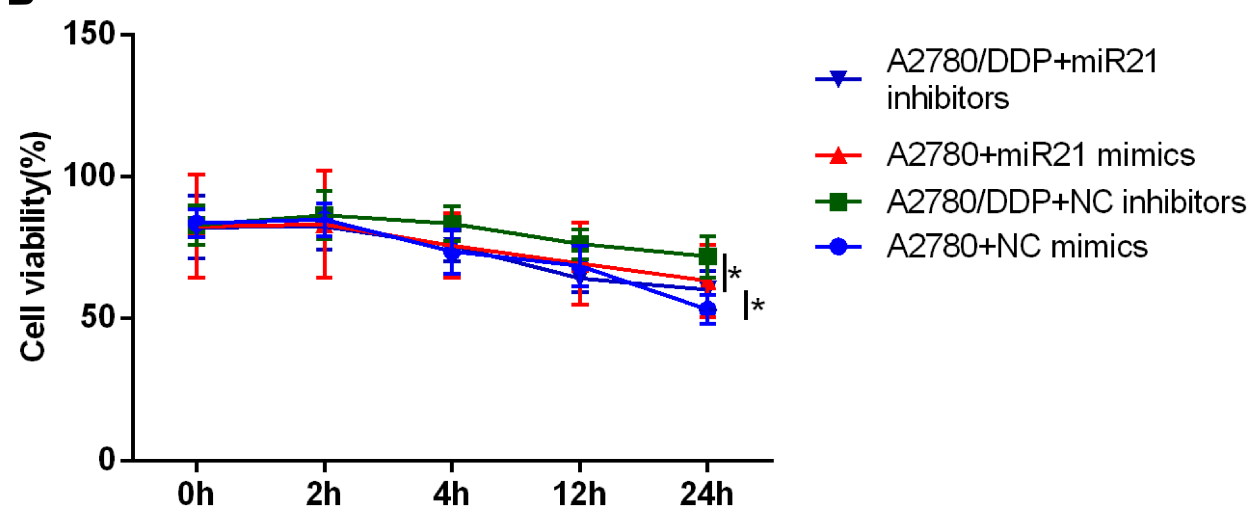

Figure 2 CCK8 assay detected cell proliferation of (A) SKOV3 and (B) A2780 at 0 h, 2 h, 4 h, 12 h, 24 h. *P<0.05; **P<0.0I.

A
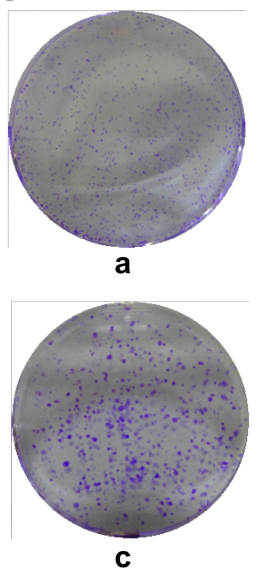

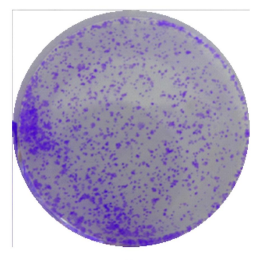

b

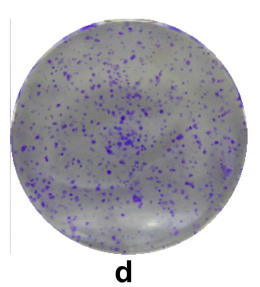

B

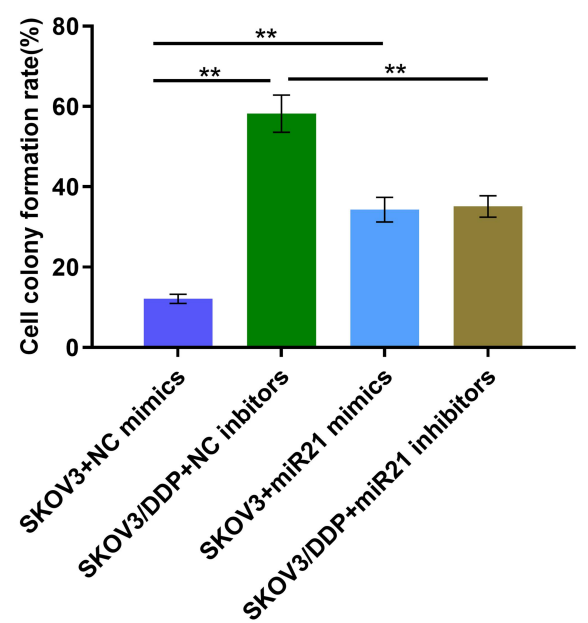

Figure 3 (A) Clonal proliferation assay of SKOV3 cells. a: SKOV3+NC mimics group; b: SKOV3/DDP+ NC inhibitors group; c: SKOV3+ miR2I mimics group; d. SKOV3/ $\mathrm{DDP}+\mathrm{miR21}$ inhibitors group. (B) Quantitative analysis of cell clone proliferation rate. $* * P<0.01$. 


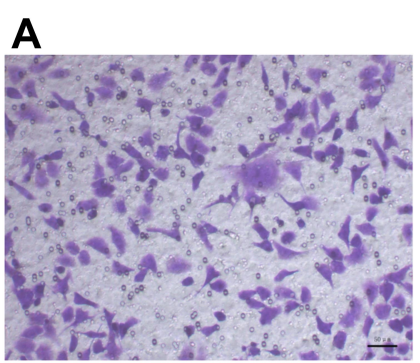

a

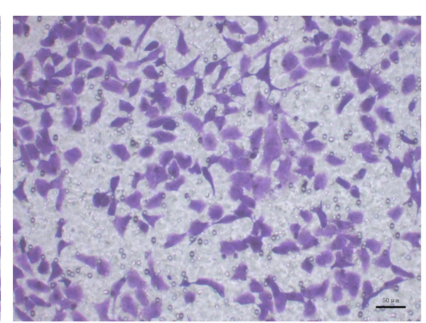

b

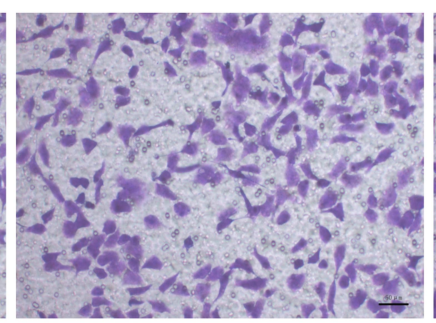

C

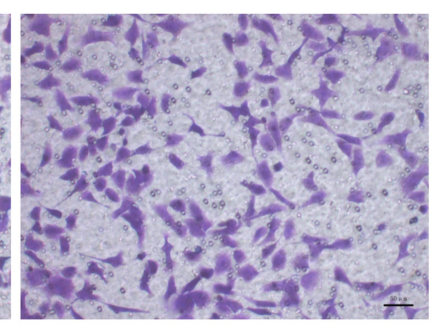

d

B

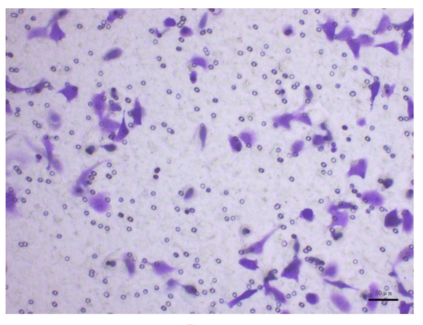

a

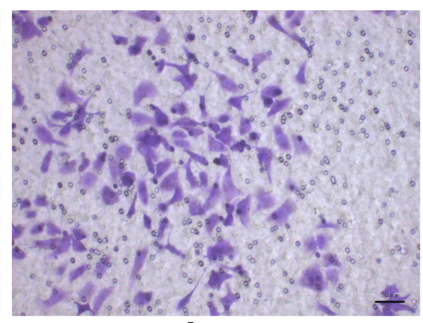

b

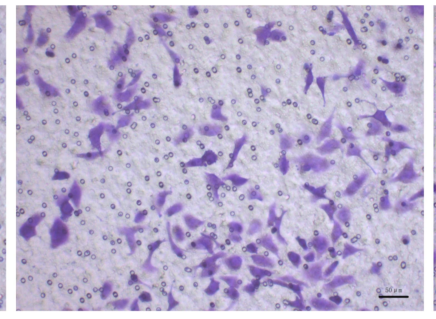

C

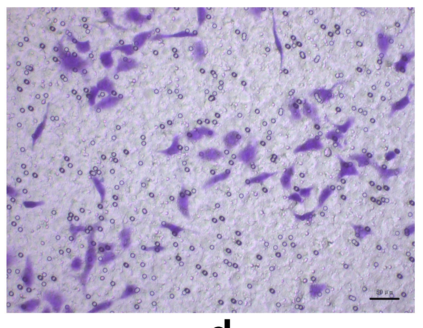

d

C
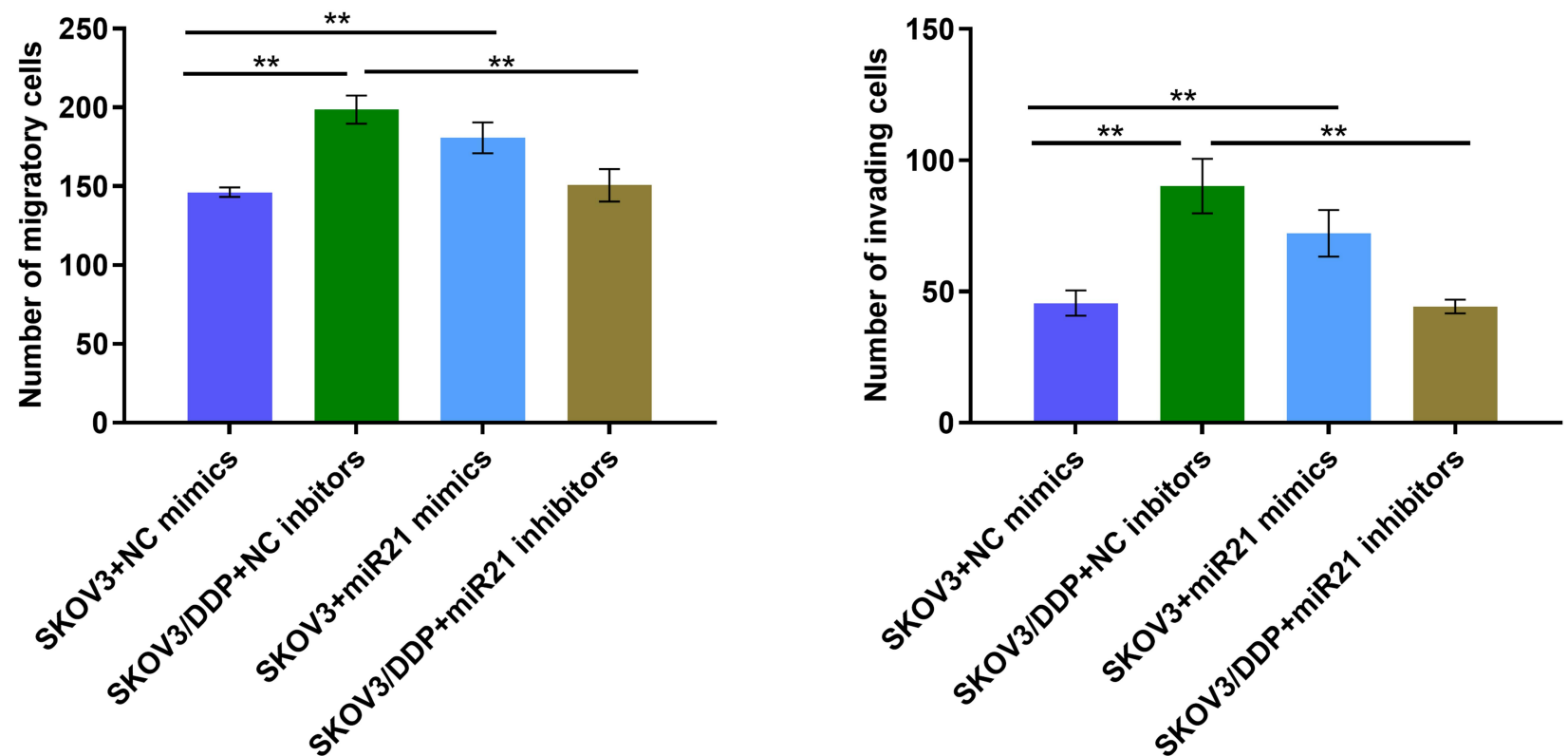

Figure 4 (A) Transwell assay detect the cell migration ability. a: SKOV3+NC mimics group; b: SKOV3/DDP+ NC inhibitors group; c: SKOV3+ miR2I mimics group; d: SKOV3/DDP+ miR2I inhibitors group. (B) Transwell assay detects the cell invasion ability. a: SKOV3+NC mimics group; b: SKOV3/DDP+ NC inhibitors group; c: SKOV3+ miR2I mimics group; d: SKOV3/DDP+ miR2I inhibitors group. (C) Quantitative analysis of migratory cells. (D) Quantitative analysis of invading cells. **P <0.01.

SKOV3/DDP cells compared that in SKOV3 cells (Figure 3, $P<0.01$ ) in mRNA and protein expression. The mRNA levels of P-gp and CD44v6 were also up-regulated in SKOV3+mi-R21 mimics group cells compared with SKOV3+NC mimics group and SKOV3+PBS group (Figure $5 \mathrm{~A}$ and $\mathrm{B}$ and $\mathrm{D}, P<0.01$ ). Western blot analysis showed that P-gp and CD44v6 protein levels were higher in SKOV3+ miR-21 mimics group cells compared with SKOV3 NC group cells (Figure 5C-E, $P<0.01$ ), which rule out the possibility that miR-21 account for the CD44v6 and P-gp higher levels in SKOV3/DDP compared with SKOV3 cells. These results suggest that miR-21 is 
A

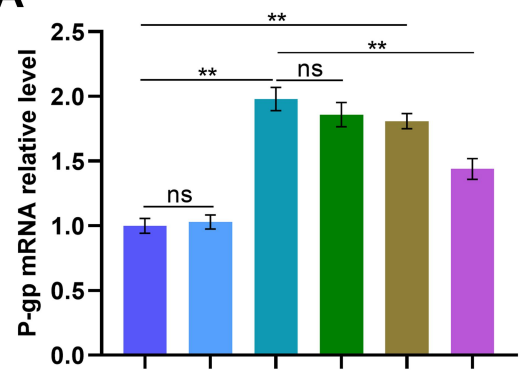

B

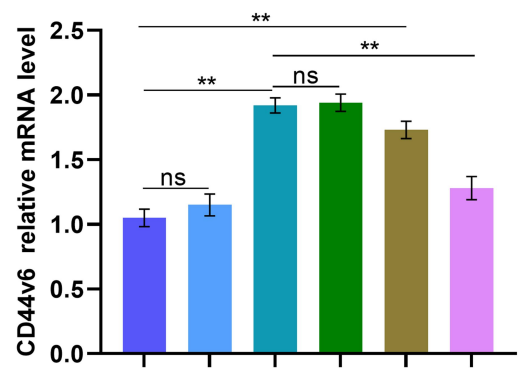

D

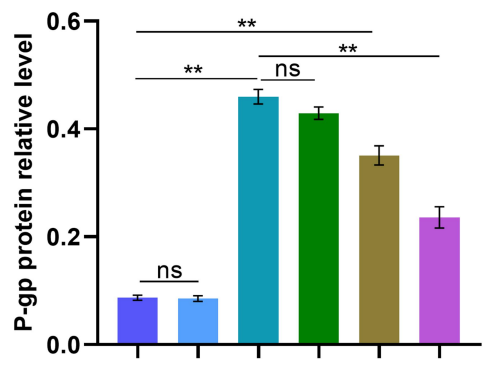

C

E

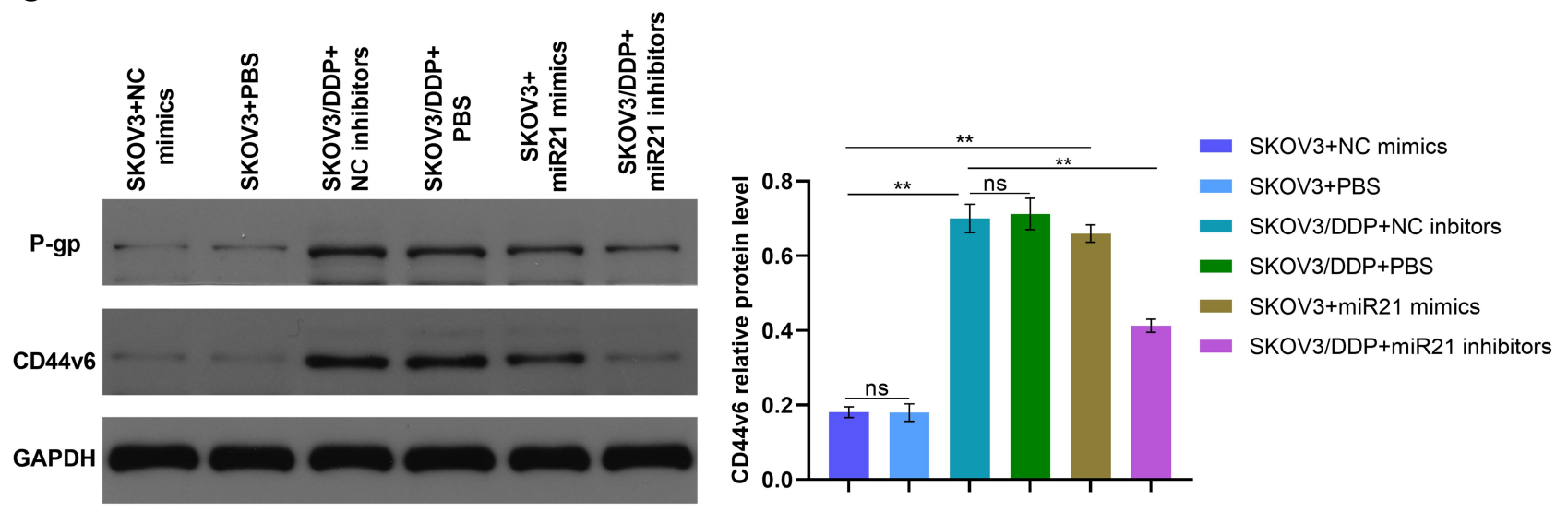

Figure 5 (A) qRT-PCR detected the P-gp relative mRNA level of SKOV3 cells transfected in miR2I mimics/inhibitors/NC/PBS. (B) qRT-PCR detected the CD44v6 relative mRNA level of SKOV3 cells transfected in miR2I mimics/inhibitors/NC/PBS. (C) The bland of P-gP and CD44v6 protein in different cells. (D) and (E) Gray value analysis of $\mathrm{P}$-gP and CD44v6 protein bands. $* * P<0.01$.

responsible for CD44v6 and P-gp expression levels in cisplatin resistant ovarian cancer cells.

\section{miR-2 I May Affect the Expression of P-gp by CD44v6}

Furthermore, to determine whether there is any interaction between CD44v6 and P-gp we transient transfected SKOV3/DDP cells with a siRNA against CD44v6. To further determine whether CD44v6 regulates cisplatin resistant cells chemotherapy resistance, we treated SKOV3/DDP-NC and SKOV3-si RNA-CD44v6 cells with cisplatin $(4.128 \mu \mathrm{M})$, Transwell assay were used for detecting cells migration, invasion ability (Figure 6A-C). When CD44v6 at low-level expression, the ability of migration and invasion also decreased significantly $(p<0.01)$. The result of Figure 6D suggest that CD44v6 has no infection on miR21 expression. Real-time PCR and Western blot analysis showed that significantly inhibited P-gp expression (Figure 6E-I, $p<0.01$ ). These results suggest that CD44v6/P-gp pathway resulted from miR-21 over-expression in cisplatin resistant ovarian cancer cells. These results suggest that CD44v6 maybe mediated miR-
21 regulation of P-gp, furthermore took part in SKOV3 cell cisplatin resistant.

\section{There May Be an Interaction Between CD44v6 and P-gP}

Since CD44v6 and P-gp are both located in the cell membrane, in order to detect the relative positions of CD44v6 and P-gp, we used immunofluorescence co-localization to detect the expression of CD44v6 and P-gp. The results showed that P-gp localized to the cytoplasm and cell membrane, and CD44v6 localized to the cell membrane, and the two proteins overlapped (Figure 7). Studies have demonstrated that P-gp expressed on the cell membrane contributes to cell resistance, and P-gp expressed in the cytoplasm has little effect on cell resistance. ${ }^{24}$ We hypothesize that there is an interaction between the protein CD44v6 and P-gp and affect the transport of the drug. We used the co-immunoprecipitation method to detect the interaction between CD44v6 and P-gp, as shown in the Figure 8.

The results suggest that there is indeed an interaction between CD44v6 and P-gp. 


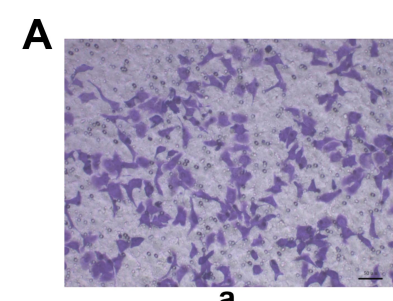

a
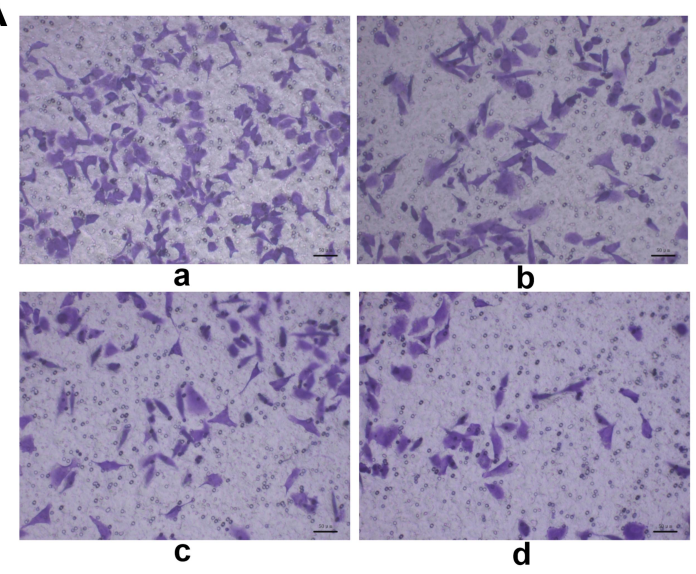

B

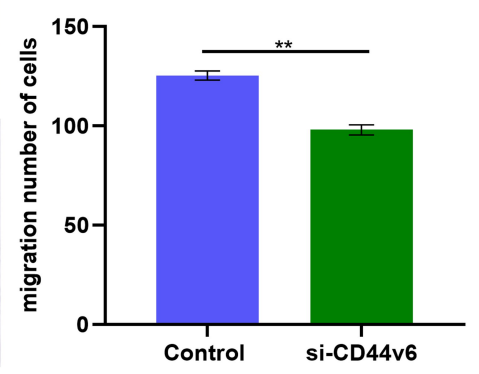

C

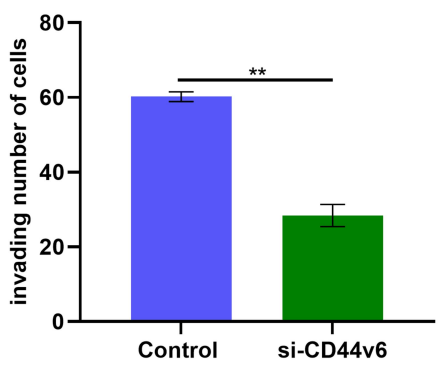

D

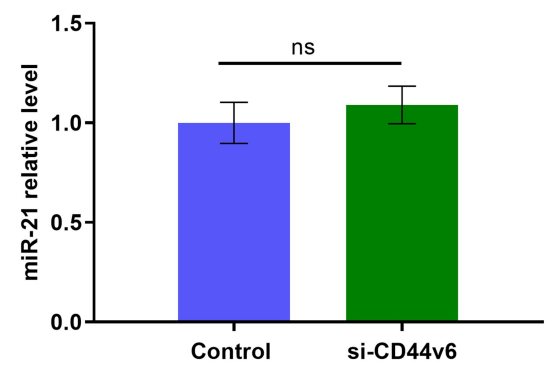

G

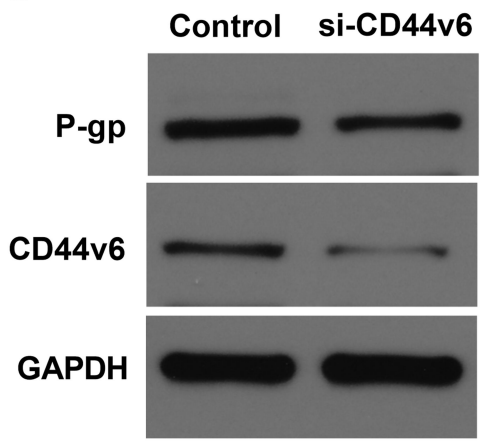

E

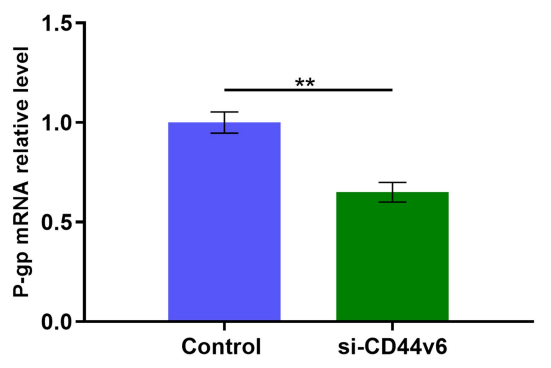

H

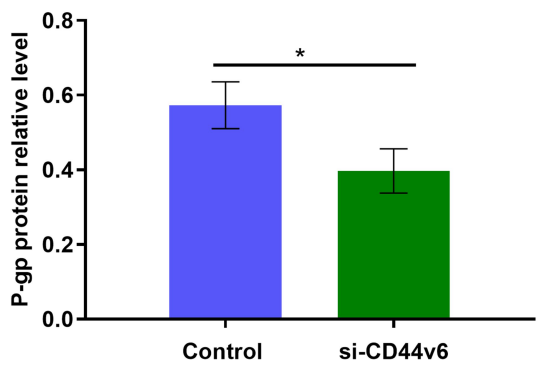

F

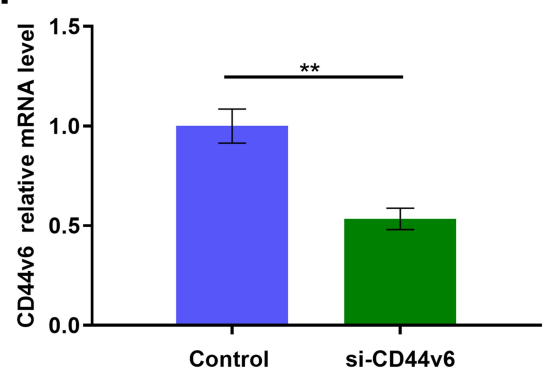

I

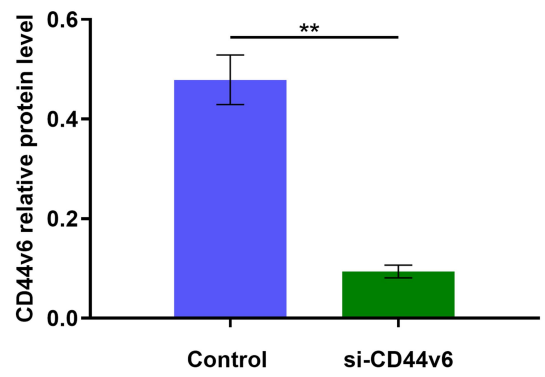

Figure 6 siRNA knockdown of CD44v6 expression in SKOV3/DDP cells. (A) Migration and invasion of SKOV3/DDP cells, a: Control group, b: si-CD44v6 group; (B) and (C)Quantification of migrating and invading cells, c: Control group, d: si-CD44v6 group; qRT-PCR and Western blot detected (D) miR-2I expression level, (E) P-gp mRNA expression level, (F) CD44v6 mRNA expression level, (G) P-gp and CD44v6 Protein expression levels. (H) and (I) Gray value analysis of CD44v6 and P-gP protein bands. $* P<0.05 ; * * P<0.01$.

\section{Discussion}

Many studies have demonstrated that miR-21 is involved in the development of chemoresistance, tumor progression and metastasis. ${ }^{17,25,26}$ In our research, we found that miR-21 can regulate the expression of CD44v6 and P-gp, which are related to OC chemoresistance and can be interact with each other.

The upregulation of miR-21 was found in direct relation with the chemoresistance of OC through the prompting of survival of cancer cells. ${ }^{27,28}$ In multiple studies of tumor chemotherapy resistance, Tominaru et $\mathrm{al}^{29}$ transfected hepatoma cells with miR-21 inhibitors to increase the sensitivity of hepatoma cells to alpha interferon/5-fluorouracil. In melanoma, inhibition of miR-21 increases the sensitivity of A357 cells and A357/CDDP cells to cisplatin. ${ }^{30}$ In the study of non-small cell lung cancer, inhibition of miR-21 expression can enhance the drug sensitivity of lung cancer-resistant cisplatin (DDP) cells A549/DDP to DDP, while overexpression of miR-21 can cause A549 cells to be resistant to chemotherapy drugs. The occurrence of medicine. 


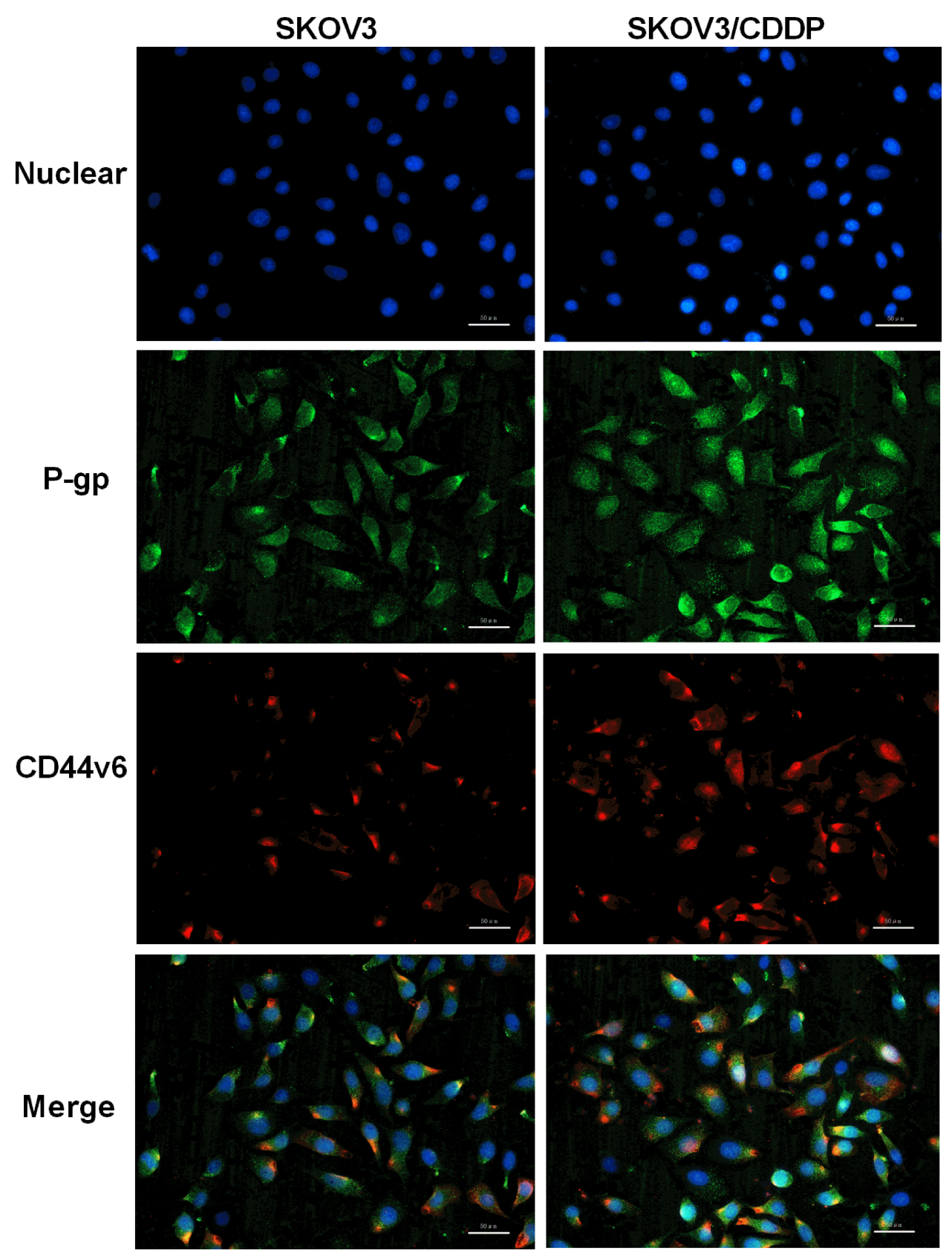

Figure 7 Immunofluorescence co-localization to detect the expression of CD44v6 and P-gp. Blue represent Nuclear, Green represent P-gp, Red represent CD44v6.

Multidrug resistance is the most common cause of tumor chemotherapy failure. Drug resistance is associated with overexpression of the drug transporter of the ATPbinding cassette protein family $(\mathrm{ABC})$, including MDR1 and its encoded products P-gp, MRP and BCRP. ${ }^{31}$ P-gp overexpression is the main cause of multidrug resistance. P-gp has an inhibitory effect on apoptosis, which establishes an organic link between tumor resistance and apoptosis tolerance at the molecular level. P-gp can delay the apoptotic cascade and protect drug-resistant cells from various forms of caspase-dependent apoptosis induced by free radicals, radiation, and cytotoxic drugs. ${ }^{32}$ To further clarify the role of miR-21 in cisplatin resistance in SKOV3 cells, miR-21 inhibitors were used to inhibit miR-21 expression in SKOV3/DDP cells. The results showed that after transfection of the miR-21 inhibitor, miR-21 expression was down-regulated in SKOV3/DDP cells, and MDR1 and its encoded protein P-gp were also downregulated relative to the negative control group. Conversely, miR-21 mimics stimulated drug resistance 


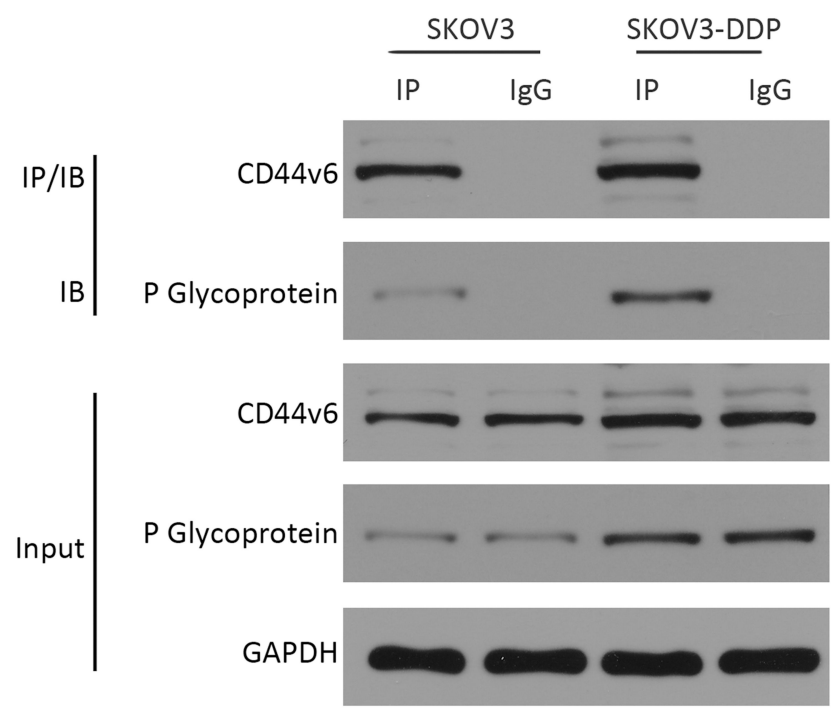

Figure 8 Co-immunoprecipitation method detected the interaction between CD44v6 and P-gp.

changes in SKOV3 cells and up-regulated the expression of MDR1 and its encoded protein P-gp.

CD44v6 is a splicing variant of the cell surface adhesion molecule CD44 family containing exon V6-encoded protein, which binds to the corresponding ligand and participates in pathological and physiological processes such as cell proliferation, apoptosis, adhesion, homing, and migration. Shi et $\mathrm{al}^{33}$ found that the serum soluble CD44v6 in patients with recurrent ovarian cancer was higher than that in patients with primary ovarian cancer. And the expression of CD44v6 in recurrent ovarian serous adenocarcinoma was significantly higher than that in patients with primary ovarian serous adenocarcinoma. Studies have shown that c-Met/ CD44v6 is associated with esophageal squamous cell carcinoma progression and chemoresistance. ${ }^{34}$ Furthermore, inhibition of miR-21 with a miR-21 specific oligonucleotide inhibitor in SKOV3/DDP cells decreased the CD44v6 and P-gp protein levels (Figure 5D), and activation of miR-21 with miR-21 mimics in SKOV3 cells increased the CD44v6 and P-gp protein levels (Figure 5D), which confirmed previous results that miR-21 regulates CD44v6 and P-gp in OC cells. Although CD44v6 and P-gp are not direct target genes of miR-21, they may be regulated indirectly by miR-21 by other factors. In addition, we found that CD44v6 expression can affect the expression of P-gp (Figure 5D), and Coimmunoprecipitation results showed that CD44v6 interacts with the p-gp protein (Figure 8), which is consistent with previous study. Miletti et $\mathrm{al}^{23}$ observed the relationships between MDR and metastatic behavior. And they found that the CD44 and P-gp has physical (ie, protein-protein) and genetic (ie, gene "cross-talk") functional interactions in cancer cells. In addition, they demonstrated that CD44 can induce P-gp expression at the mRNA level and influence cancer cell invasion. ${ }^{23}$ Misra et al have reported that CD44 regulates phosphoinositide 3-kinase activity, which is participated in the stimulation of P-gp expression. ${ }^{35}$

In summary, our study transfected human OC cell SKOV3 with miR-21 mimics, transfected SKOV3/DDP cells with miR-21 inhibitors, and found that miR-21 may affect ovarian cancer cells platinum resistance by regulating CD44v6 and P-gp expression. Furthermore, we knocked down CD44v6 in OC SKOV3/DDP cells and found that CD44v6 can mediated miR-21 regulating P-gp expression. In addition, since both CD44v6 and P-gp are localized in the cell membrane, we confirmed that the two proteins CD44v6 and P-gp can physically interact with each other by CO-IP. Therefore, miR-21/CD44v6/P-gp may be a potential target for reversing the resistance of cisplatin chemotherapy in $\mathrm{OC}$, which is of great development and application value. However, there are some shortcomings in our research for that the absence of normal cells as negative controls in our experiments is a limitation of our study. And the relationship between miRNA21, CD44v6, and P-gp need more experiments. Furthermore, we still lack in vivo animal experiments to confirm our results.

\section{Ethics Approval}

No ethics issues in this study.

\section{Acknowledgments}

The study was supported by the Independent Research Project of Wuhan University (grant no. 413000117) and the Special Fund for Clinical Medicine Research of Chinese Medical Association (grant no. 17020310700).

\section{Disclosure}

The authors report no conflicts of interest in this work.

\section{References}

1. Zhang X-Y, Zhang P-Y. Recent perspectives of epithelial ovarian carcinoma. Oncol Lett. 2016;12(5):3055-3058. doi:10.3892/ ol.2016.5107

2. Karimi-Zarchi M, Mortazavizadeh SM, Bashardust N, et al. The clinicopathologic characteristics and 5-year survival rate of epithelial ovarian cancer in Yazd, Iran. Electron Physician. 2015;7(6):13991406. doi:10.14661/1399

3. Zhang X-Y, Zhang PY. Recent perspectives of epithelial ovarian carcinoma. Oncol Lett. 2020;20(5). 
4. Siegel RL, Miller KD, Jemal A. Cancer statistics, 2018. CA Cancer J Clin. 2018;68(1):7-30. doi:10.3322/caac.21442

5. Chen W, Zheng R, Baade PD, et al. Cancer statistics in China, 2015. CA Cancer J Clin. 2016;66(2):115-132. doi:10.3322/caac.21338

6. Siegel RL, Miller KD, Jemal A. Cancer statistics, 2015. CA Cancer J Clin. 2015;65(1):5-29. doi:10.3322/caac. 21254

7. Au Yeung CL, Co NN, Tsuruga T, et al. Exosomal transfer of stromaderived miR21 confers paclitaxel resistance in ovarian cancer cells through targeting APAF1. Nat Commun. 2016;7:11150. doi:10.1038/ ncomms 11150

8. Lloyd KL, Cree IA, Savage RS. Prediction of resistance to chemotherapy in ovarian cancer: a systematic review. BMC Cancer. 2015;15:117. doi:10.1186/s12885-015-1101-8

9. Cannistra SA. Cancer of the ovary. N Engl J Med. 2004;351 (24):2519-2529. doi:10.1056/NEJMra041842

10. Liu LL, Lu SX, Li M, et al. FoxD3-regulated microRNA-137 suppresses tumour growth and metastasis in human hepatocellular carcinoma by targeting AKT2. Oncotarget. 2014;5(13):5113-5124. doi:10.18632/oncotarget.2089

11. Yanaihara N, Caplen N, Bowman E, et al. Unique microRNA molecular profiles in lung cancer diagnosis and prognosis. Cancer Cell. 2006;9(3):189-198. doi:10.1016/j.ccr.2006.01.025

12. Fiannaca A, La Rosa M, La Paglia L, et al. Analysis of miRNA expression profiles in breast cancer using biclustering. $B M C$ Bioinform. 2015;16(Suppl 4):S7. doi:10.1186/1471-2105-16-S4-S7

13. Lee EJ, Gusev Y, Jiang J, et al. Expression profiling identifies microRNA signature in pancreatic cancer. Int J Cancer. 2007;120 (5):1046-1054. doi:10.1002/ijc.22394

14. Ruan K, Fang X, Ouyang G. MicroRNAs: novel regulators in the hallmarks of human cancer. Cancer Lett. 2009;285(2):116-126. doi:10.1016/j.canlet.2009.04.031

15. Vandghanooni S, Eskandani M, Montazeri V, et al. SurvivindeltaEx3: a novel biomarker for diagnosis of papillary thyroid carcinoma. J Cancer Res Ther. 2011;7(3):325-330. doi:10.4103/09731482.87038

16. Dong Z, Ren L, Lin L, et al. Effect of microRNA-21 on multidrug resistance reversal in A549/DDP human lung cancer cells. Mol Med Rep. 2015;11(1):682-690. doi:10.3892/mmr.2014.2662

17. Pink RC, Samuel P, Massa D, et al. The passenger strand, miR-21-3p, plays a role in mediating cisplatin resistance in ovarian cancer cells. Gynecol Oncol. 2015;137(1):143-151. doi:10.1016/j.ygyno.2014.12.042

18. Echevarria-Vargas IM, Valiyeva FandVivas-Mejia PE. Upregulation of miR-21 in cisplatin resistant ovarian cancer via JNK-1/c-Jun pathway. PLoS One. 2014;9(5):e97094. doi:10.1371/journal. pone.0097094

19. Yang H, Kong W, He L, et al. MicroRNA expression profiling in human ovarian cancer: miR-214 induces cell survival and cisplatin resistance by targeting PTEN. Cancer Res. 2008;68(2):425-433. doi:10.1158/0008-5472.CAN-07-2488

20. Xie Z, Cao L, Zhang J. miR-21 modulates paclitaxel sensitivity and hypoxia-inducible factor-1alpha expression in human ovarian cancer cells. Oncol Lett. 2013;6(3):795-800. doi:10.3892/ol.2013.1432

21. Morath I, Hartmann TN, Orian-Rousseau V. CD44: more than a mere stem cell marker. Int J Biochem Cell Biol. 2016;81(Pt A):166-173. doi:10.1016/j.biocel.2016.09.009

OncoTargets and Therapy

\section{Publish your work in this journal}

OncoTargets and Therapy is an international, peer-reviewed, open access journal focusing on the pathological basis of all cancers, potential targets for therapy and treatment protocols employed to improve the management of cancer patients. The journal also focuses on the impact of management programs and new therapeutic

Submit your manuscript here: https://www.dovepress.com/oncotargets-and-therapy-journal
22. Ravindranath AK, Kaur S, Wernyj RP, et al. CD44 promotes multidrug resistance by protecting P-glycoprotein from FBXO21-mediated ubiquitination. Oncotarget. 2015;6(28):26308-26321. doi:10.18632/ oncotarget. 4763

23. Miletti-Gonzalez KE, Chen S, Muthukumaran N, et al. The CD44 receptor interacts with P-glycoprotein to promote cell migration and invasion in cancer. Cancer Res. 2005;65(15):6660-6667. doi:10.1158/0008-5472.CAN-04-3478

24. Qin Y, Zhang Z, Huang C, et al. Folate-targeted redox-responsive polymersomes loaded with chemotherapeutic drugs and tariquidar to overcome drug resistance. J Biomed Nanotechnol. 2018;14 (10):1705-1718. doi:10.1166/jbn.2018.2623

25. Baez-Vega PM, Echevarria Vargas IM, Valiyeva F, et al. Targeting miR-21-3p inhibits proliferation and invasion of ovarian cancer cells. Oncotarget. 2016;7(24):36321-36337. doi:10.18632/oncotarget.9216

26. Chen Y, Chen Q, Liu Q, et al. Human epididymis protein 4 expression positively correlated with miR-21 and served as a prognostic indicator in ovarian cancer. Tumour Biol. 2016;37(6):8359-8365. doi:10.1007/s13277-015-4672-8

27. Feng YHandTsao Y-H, Tsao C-J. Emerging role of microRNA-21 in cancer. Biomed Rep. 2016;5(4):395-402. doi:10.3892/br.2016.747

28. Bai H, Xu R, Cao Z, et al. Involvement of miR-21 in resistance to daunorubicin by regulating PTEN expression in the leukaemia K562 cell line. FEBS Lett. 2011;585(2):402-408. doi:10.1016/j. febslet.2010.12.027

29. Tomimaru Y, Eguchi H, Nagano H, et al. MicroRNA-21 induces resistance to the anti-tumour effect of interferon-alpha/5-fluorouracil in hepatocellular carcinoma cells. Br J Cancer. 2010;103(10):16171626. doi:10.1038/sj.bjc. 6605958

30. Zhang HL, Si LB, Zeng A, et al. MicroRNA-21 antisense oligonucleotide improves the sensitivity of A375 human melanoma cell to Cisplatin: an in vitro study. J Cell Biochem. 2018;119(4):3129-3141. doi: $10.1002 / \mathrm{jcb} .26455$

31. El-Readi MZ, Eid S, Abdelghany AA, et al. Resveratrol mediated cancer cell apoptosis, and modulation of multidrug resistance proteins and metabolic enzymes. Phytomedicine. 2019;55:269-281. doi:10.1016/j.phymed.2018.06.046

32. Guo Q, Jing FJ, Qu HJ, et al. Ubenimex reverses MDR in gastric cancer cells by activating caspase-3-mediated apoptosis and suppressing the expression of membrane transport proteins. Biomed Res Int. 2019;2019:4390839. doi:10.1155/2019/4390839

33. Shi J, Zhou Z, Di W, Li N. Correlation of CD44v6 expression with ovarian cancer progression and recurrence. BMC Cancer. 2013,13:182. doi:10.1186/1471-2407-13-182

34. Hara T, Makino T, Yamasaki M, et al. Effect of c-Met and CD44v6 expression in resistance to chemotherapy in esophageal squamous cell carcinoma. Ann Surg Oncol. 2019;26(3):899-906. doi:10.1245/ s10434-018-07126-5

35. Misra S, Ghatak S, Toole BP Regulation of MDR1 expression and drug resistance by a positive feedback loop involving hyaluronan, phosphoinositide 3-kinase, and ErbB2. J Biol Chem. 2005;280 (21):20310-20315. doi:10.1074/jbc.M500737200 agents and protocols on patient perspectives such as quality of life, adherence and satisfaction. The manuscript management system is completely online and includes a very quick and fair peer-review system, which is all easy to use. Visit http://www.dovepress.com/ testimonials.php to read real quotes from published authors. 